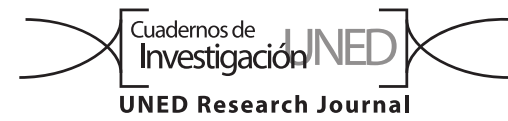

\title{
Tradición: una nueva razón para alimentar las palomas urbanas (Columba livia; Columbiformes: Columbidae), y cómo controlarlas de manera sostenible
}

\author{
Andrea Cabalceta' \& Zaidett Barrientos² \\ 1. Investigadora independiente, Urbanización Gloria Bejarano, Tirrases, Curridabat, Costa Rica; \\ andreacab1982@hotmail.com, (iD https://orcid.org/0000-0002-3654-6262 \\ 2. Universidad Estatal a Distancia, Vicerrectoría de Investigación, Laboratorio de Ecología Urbana, San José, Costa Rica; \\ zaidettbarrientos@gmail.com, (iD https://orcid.org/0000-0001-5608-6139 \\ Recibido 04-V-2019 • Corregido 19-VII-2019 • Aceptado 30-VII-2019 \\ DOI: https://doi.org/10.22458/urj.v11i3.2216
}

\begin{abstract}
Tradition: a new reason to feed urban pigeons (Columba livia, Columbiformes: Columbidae), and how to control them in a sustainable way". Introduction: The rock pigeon, Columba livia, is considered a pest in many cities around the world, where people feed them in parks and control measures have mostly failed; however, they also provide entertainment and income to people. Objective: To identify the reasons why humans feed pigeons, the type of food and their perception of the problem. Methods: We worked in eight urban recreation parks of Costa Rica (total: 59 visits). From July to September 2017 we interviewed 161 people feeding pigeons and 90 who were in the parks but did not feed them. Results: People fed them scraps from their own food or, preferably, if they were sold for the purpose, seeds. The reason why people said they fed pigeons was independent of age, academic level and visit purpose (contingency chi square tests, all: $p>0,01$ ). One of the reasons was tradition; other reasons were "for fun" and empathy; men were more focused on empathy than women. Most people who fed pigeons did not consider them a pest and were uncertain about any need for control. Non feeders mostly considered them pests that need to be controlled. Almost everyone found contraceptives acceptable. Conclusions: A sustainable strategy for urban pigeons would include controlled food sales and contraception, instead of prohibition or extermination; this is the first time that tradition is found as a reason for feeding pigeons anywhere in the world.
\end{abstract}

Key words: Feeding of birds, informal sales, tradition, social perception.

La Paloma de Castilla, Columba livia, habitaba, originalmente, en acantilados costeros alrededor del mediterráneo europeo, africano y asiático, pero actualmente se distribuye por casi todo el mundo (Stringham et al., 2012; Stock \& Haag-Wackernage, 2014). Las construcciones en las ciudades ofrecen oportunidades de anidación,
Resumen: Introducción: La Paloma de Castilla, Columba livia, es considerada una plaga en muchas ciudades del mundo. En las ciudades las personas las alimentan y las medidas de control han fallado, sin embargo, ellas también dan entretenimiento e ingresos económicos a las personas. Objetivo: Identificar las razones porque las personas alimentan a las aves, el tipo de comida que les dan y su percepción del problema. Métodos: Trabajamos en ocho parques urbanos de Costa Rica. Entre julio y setiembre del 2017 encuestamos 161 personas que alimentaban a las palomas y 90 que estaban en los parques urbanos, pero no las alimentaban. Resultados: Las personas alimentaban a las palomas con sobros de su comida o preferiblemente con semillas, si estaban a la venta en las cercanías. La razón por la que la gente decía que alimentaba a las palomas fue independiente de la edad, la escolaridad y el propósito de la visita al parque (chi cuadrado de contingencia, todos: $p>0,01$ ). Una de las razones para alimentarlas fue tradición; otras razones fueron diversión y empatía; los hombres lo hacían más por empatía que las mujeres. La mayor parte de las personas que alimentaban a las palomas consideran que no son una plaga que necesite ser controlada. Los que no las alimentaban consideraban que son una plaga que debe ser controlada. Casi todos consideraron el uso de anticonceptivos como una medida de control aceptable. Conclusiones: Una estrategia sostenible para el manejo de palomas urbanas debe incluir ventas controladas de alimento para aves y el uso de anticonceptivos, en vez de prohibir que se les alimento y que se promueva su exterminio; esta es la primera vez que se encuentra la tradición como una razón para alimentar a las palomas.

Palabras clave: Alimentación de aves, ventas informales; tradición; percepción social. reproducción y descanso similares a las que ofrecen los acantilados de donde provienen (Spennemann \& Watson, 2017).

En su hábitat natural estas aves son principalmente granívoras, aunque ocasionalmente pueden ingerir invertebrados (Murton \& Westwood, 1966; Spennemann 
\& Watson, 2017). Por el contrario, en las ciudades, se alimentan principalmente de la comida que las personas les proporcionan (Ramírez et al., 2008; Ryan, 2011; Spennemann \& Watson, 2017). Estos alimentos, generalmente cargados de carbohidratos, azúcares y condimentos, se alejan de la dieta original de la especie provocando desnutrición, peso reducido y un porcentaje de grasa corporal elevado (Municipalidad de Heredia, 2013; Spennemann \& Watson, 2017). Además, en la naturaleza, al contrario de las zonas urbanas, el alimento es escaso y no se acumula en lugares puntuales (Ryan, 2011), por lo que las aves se ejercitan al buscarlo, mejorando su proporción corporal de grasas y proteínas (Ciminari, Moyano, Chediack, \& Caviedes-Vidal, 2005).

En muchos países el manejo de la Paloma de Castilla en zonas urbanas ha enfatizado la importancia de evitar que las personas las alimenten (Ryan, 2011; Mondocorre, Pérez, Albis, \& Zeballos, 2014; Stock \& Haag-Wackernagel, 2014; Senar, Montalvo, Pascual, \& Peracho, 2016). Sin embargo, el éxito de las campañas en contra de alimentarlas es limitado (Senar et al., 2016) y no se conoce la opinión de los visitantes de parques urbanos tropicales sobre las razones que tienen para alimentarlas, ni de si las consideran una plaga.

Los principales métodos de control que se han utilizado para regular las poblaciones de la Paloma de Castilla se dividen en cuatro grupos: 1 ) sacrificio de animales; 2 ) disminución del éxito reproductivo mediante remoción de nidos y uso de sustancias anticonceptivas; 3 ) disminución de la capacidad de carga del hábitat mediante el control de la cantidad de alimento, la instalación de barreras físicas para que no aniden y la utilización de repelentes químicos, sonoros y visuales; y 4) captura y traslado de las aves (Giunchi, Albores-Barajas, Baldaccini, Vanni, \& Soldatini, 2012; Stock \& Haag-Wackernagel, 2014). No se ha estudiado la opinión del público sobre la aplicación de estas medidas.

El estado de salud de las Palomas de Castilla en Costa Rica es deficiente debido principalmente a una mala alimentación (Municipalidad de Heredia, 2013). Además, desde el 2010, el tamaño de su población en parques urbanos ha aumentado tanto que ha sido necesaria algún tipo de intervención (Gutiérrez, 2010; Artavia, 2017). Algunas de las medidas que se han implementado son el uso de modelos de aves rapaces, la emisión de sonidos para ahuyentarlas, la poda de árboles, el envenenamiento masivo, la remoción de huevos, las mallas para evitar que aniden y la remodelación de infraestructura (Gutiérrez, 2010; La Nación, 2013; Cabezas, 2013; Repretel, 2016). Todas esas actividades se han realizado sin contar con un verdadero plan de manejo de la especie a nivel nacional, por lo tanto, el tamaño de las poblaciones de palomas solo ha disminuido temporalmente. Ante el fracaso de los diferentes métodos utilizados, se ha recurrido a medidas paliativas para disminuir el contacto de las personas con las palomas: traslado de estaciones de transporte, suspensión de actividades recreativas, cívicas y culturales en el parque (Arroyo, 2017; Astorga, 2019; Fernández, 2019). Desde el 2018, se está planeando probar con sustancias anticonceptivas de una forma más sistematizada en varios parques urbanos, sin embargo, los trámites de permisos para la importación de esas sustancias han retrasado el proyecto (Lynch, 2018; Multimedios, 2018).

Las hipótesis de investigación de este trabajo son: 1) las ventas informales de alimento para aves favorecen que las palomas urbanas se alimenten de granos y semillas; 2 ) las personas que alimentan a las palomas lo hacen más por diversión que por tradición, empatía con las aves, llamar la atención de otras personas o establecer relaciones interpersonales con otros aficionados a las aves; 3 ) el motivo por el que alimentan a las palomas es independiente de la edad, sexo, nivel de escolaridad y objetivo de la visita al parque; 4) las personas que alimentan a las palomas no las perciben como una plaga y no aceptan el uso de métodos de control de la población; 5) la mayoría de las personas, indistintamente de si las alimentan o no, consideran aceptable el uso de sustancias anticonceptivas para palomas.

\section{METODOLOGÍA}

El trabajo de campo lo realizamos entre julio y setiembre del 2017 en ocho parques urbanos de San José, Costa Rica (Cuadro 1, Apéndice digital: Ubicación geográfica de los sitios estudiados). Los parques seleccionados miden entre 1755 y $8292 m^{2}$ y están rodeados de infraestructura urbana, como iglesias, comercios y edificios. Además, todos tienen Palomas de Castilla, topografía plana, paradas de transporte público, zonas con pasto y árboles, y estructuras de cemento tales como: bancas, fuentes de agua o lagos artificiales, zonas asfaltadas, kioscos, monumentos, anfiteatros y juegos para niños. En cuanto al cli$\mathrm{ma}$, se trata de una zona urbana que originalmente tuvo un bosque tropical premontano, con una temperatura promedio de $20,3^{\circ} \mathrm{C}$ y una precipitación anual de entre 1500 y $2000 \mathrm{~mm}$ (Holdridge, Grenke, Hatheway, Liang, \& Tosi, 1971; Monge-Nájera, Gómez, \& Rivas, 2008). Los parques urbanos los clasificamos según la presencia o ausencia de ventas informales de alimento para aves, en las cuales vendían paquetes de aproximadamente $60 \mathrm{~g}$ de maíz crudo para consumo humano (Cuadro 1). Los muestreos los realizamos entre las 9:00am y las 12:00m y 
CUADRO 1

Parques urbanos estudiados, San José, Costa Rica

\begin{tabular}{lcccc}
\multicolumn{1}{c}{ Nombre del parque urbano } & $\begin{array}{c}\text { Área total } \\
\left(\mathrm{m}^{2}\right)\end{array}$ & $\begin{array}{c}\text { Porcentaje del área con } \\
\text { cobertura de cemento }\end{array}$ & $\begin{array}{c}\text { Área de cobertura vegetal } \\
\text { en vista aérea }\left(\mathrm{m}^{2}\right)^{*}\end{array}$ & $\begin{array}{c}\text { Ventas informales de } \\
\text { alimento para aves }\end{array}$ \\
Plaza Juan Mora Fernández & 1755 & 92 & 479 & Sí \\
Parque Central & 6610 & 89 & 2426 & Sí \\
Parque de Guadalupe & 7911 & 43 & 3458 & Sí \\
Boulevar del Correo & 1988 & 82 & 670 & No \\
Parque de las Garantías Sociales & 6008 & 86 & 2420 & No \\
Parque La Paz ** & 5646 & 44 & 3890 & No \\
Parque Okayama & 8292 & 28 & 6129 & No \\
Parque Sabanilla & 2840 & 14 & 2429 & No \\
\hline
\end{tabular}

* Incluye el área cubierta por zonas de zacate y copas de árboles.

** Este parque mide 53ha, sin embargo, solo trabajamos en la sección en donde se concentran las palomas.

entre las 13:00 pm a las 16:00 pm. Únicamente la primera autora participó en la recolección de datos.

Visitamos cada parque aproximadamente siete veces para un total de 59 visitas, un tercio las realizamos en fin de semana. En el estudio consideramos únicamente personas de 18 años o más, les comentamos los objetivos del proyecto y les solicitamos su anuencia para participar en el estudio. Aplicamos 161 encuestas a personas que estaban alimentando a las palomas y 90 a personas que no las alimentaban.

Solamente a los encuestados que estaban alimentando a las palomas se les preguntó por el tipo de alimento que les daban y la razón por la que lo hacían (Apéndice digital: Encuestas). Los datos sobre el tipo de alimento que les brindan a las palomas se analizó considerando si en el parque había ventas informales de comida para aves.

Tanto a los encuestados que alimentaban a las palomas como a los que no las alimentaban les consultamos sobre su percepción acerca de si las palomas son plaga y si se debe controlar la población y sobre los métodos de control que se pueden aplicar para regular las poblaciones (Apéndice digital: Encuestas).

No limitamos la cantidad de alimentos que podía mencionar cada persona, por lo tanto, la suma de tipo de alimentos es mayor a la cantidad de personas encuestadas. A cada alimento mencionado por los encuestados le asignamos el valor de uno. Clasificamos los alimentos mencionados en cuatro categorías considerando el valor nutricional para una especie granívora: 1) granos crudos, esta categoría incluye principalmente maíz, pero también arroz, alpiste y concentrado para patos; 2 ) residuos de comida, incluye granos cocidos, galletas y otros alimentos usuales en la dieta humana; 3) alimentos procesados, incluye alimentos de consumo humano ricos en azúcar y condimentos tales como plátanos, papas, yuca y maíz en hojuelas o láminas, fritos, salados, dulces y/o condimentados; y 4) otros, esta categoría incluye alimento que las palomas pueden recolectar por búsqueda, por ejemplo, insectos y semillas de la vegetación del parque. Los datos los analizamos según hubiera o no ventas informales en el parque.

Las razones por las que las personas dan alimento a las palomas las agrupamos en cuatro categorías: 1) diversión, cuando las personas alimentan a las palomas por entretenimiento o distracción; 2) tradición, cuando las alimentan por costumbre, generalmente transmitida por generaciones; 3) empatía, cuando expresaban admiración, compasión, identificación o amistad por las palomas; y 4) mixto, cuando mencionaban dos o tres de las categorías anteriores. El motivo de la visita lo categorizamos en: recreación, tránsito y otro. La edad la categorizamos de 18 a 29 años, de 30 a 49 y en 50 o más años. Las categorías de escolaridad que utilizamos fueron: 1) ninguna educación o primaria completa o incompleta, 2) secundaria completa o incompleta y 3 ) universidad completa o incompleta.

En todos los casos (Cuadros 2 a 6) aplicamos pruebas de chi cuadrado de bondad de ajuste dentro de cada columna (el resultado siempre fue $p<0,0001$ ) y luego una prueba adicional de contingencia al cuadro completo, que es la que se detalla al pie.

\section{RESULTADOS}

Efecto de las ventas informales en el tipo de alimento que ingieren las palomas (hipótesis 1) 
En los lugares donde hay ventas informales de alimento para palomas la mayoría de las personas dicen que dan granos crudos a las palomas (Cuadro 2). Por el contrario, donde no hay ventas informales de comida para palomas, la mayoría dicen que les dan residuos de comida para humanos (Cuadro 2).

\section{Razón por la que las personas alimentan a las palomas (hipótesis 2 y 3 )}

Las personas que alimentan a las palomas lo hacen principalmente por diversión $(33 \%, \mathrm{n}=161)$ y empatía (35\%, $\mathrm{n}=161$ ) (Chi cuadrado de bondad de ajuste: $\mathrm{x}^{2}=$ $25,41 ; g l=3 ; p<0,0001)$. No obstante, el porcentaje de personas que lo hacen por tradición es bastante alto $(21 \%, \mathrm{n}=161)$.

La razón por la que alimentan a las palomas es independiente de la edad (Chi cuadrado de bondad de ajuste $x^{2}=8,1 ; g l=6 ; p=0,2309$ ), del nivel de escolaridad (Chi cuadrado de bondad de ajuste: $\left.x^{2}=1,55 ; g l=6 ; p=0,9561\right)$ y del objetivo de la visita al parque (Chi cuadrado de bondad de ajuste: $\left.x^{2}=12,5, g l=6 ; p=0,0517\right)$. Únicamente encontramos que el sexo de las personas afecta la razón por la cual alimentan a las palomas (Cuadro 3). Las mujeres alimentan a las palomas en igual proporción por tradición, diversión y empatía. Por el contrario, los hombres las alimentan principalmente por empatía.

\section{Relación entre la costumbre de alimentar a las palomas y la percepción de la especie como plaga (hipótesis 4)}

Al contrario de las personas que no alimentan a las palomas, las personas que sí las alimentan tienden a considerar que las palomas no son una plaga y son más cautelosos al expresar su aprobación a la aplicación de métodos de control (Cuadros 4 y 5).

\section{Percepción del uso de métodos de control de poblaciones de palomas de acuerdo a la costumbre de alimentarlas o no alimentarlas (hipótesis 5)}

El uso de sustancias anticonceptivas fue el método más aceptado tanto por las personas que alimentan a las palomas como por las que no (Cuadro 6). En ambos casos, el porcentaje de personas que no aceptan ningún tipo de control es relativamente bajo. Sin embargo, como es de esperar, es mayor en las que no las alimentan (Cuadro 6). Además, las personas que no alimentan a las palomas aceptan bien la remoción de nidos.

CUADRO 2

Tipo de alimentos que las personas dicen darle a las palomas en parques urbanos de San José, Costa Rica

\begin{tabular}{lcccc}
\multicolumn{1}{c}{$\%$} & Granos crudos & Residuos de comida & Alimentos procesados & Otros \\
$\begin{array}{l}\text { Parque urbano con ventas de alimento } \\
\text { para palomas }(\mathrm{n}=71)\end{array}$ & 54 & 21 & 20 & 5 \\
$\begin{array}{l}\text { Parque urbano sin ventas de alimento } \\
\text { para palomas }(\mathrm{n}=120)\end{array}$ & 21 & 65 & 12 & 2 \\
\hline
\end{tabular}

(Chi cuadrado de contingencia: $\left.x^{2}=35,25 ; g l=3 ; p<0,01\right)$.

\section{CUADRO 3}

Razones sociales para alimentar palomas en zonas de recreo urbanos de San José, Costa Rica, según el género de los encuestados que las alimentan sin fines de lucro

\begin{tabular}{ccccc} 
\% & Tradición & Diversión & Empatía & Mixto \\
Mujeres $(n=132)$ & 18,6 & 29,2 & 24,2 & 10 \\
Hombres $(n=29)$ & 2,5 & 3,7 & 11,2 & 0,6 \\
\hline
\end{tabular}

(Chi cuadrado de contingencia: $\left.x^{2}=11,3 ; g l=3 ; p<0,01\right)$. 


\section{CUADRO 4}

Percepción de personas que alimentan a las palomas y de visitantes que no las alimentan sobre si las palomas son plaga

\begin{tabular}{lcccc} 
\% & Si son plaga & No son plaga & Indeciso & No sabe \\
Personas que alimentan a las palomas $\mathrm{n}=161$ & 13 & 65 & 15 & 7 \\
Personas que no alimentan a las palomas $\mathrm{n}=90$ & 45 & 23 & 23 & 9 \\
\hline
\end{tabular}

(Chi cuadrado de contingencia: $X^{2}=45,59, g l=3 ; p<0,01$ ).

\section{CUADRO 5}

Opinión de las personas que alimentan a las palomas y de las que no las alimentan sobre si se deben aplicar métodos de control de la población de palomas

\begin{tabular}{ccccc} 
\% & $\begin{array}{c}\text { Si se debe aplicar } \\
\text { métodos de control }\end{array}$ & $\begin{array}{c}\text { No se deben aplicar } \\
\text { métodos de control }\end{array}$ & $\begin{array}{c}\text { Indeciso } \\
\text { No sabe }\end{array}$ \\
Personas que alimentan a las paloma $n=161$ & 13 & 25 & 55 & 7 \\
Personas que no alimentan a las paloma $n=90$ & 52 & 11 & 36 \\
\hline
\end{tabular}

(Chi cuadrado de contingencia: $\left.X^{2}=47,06, g l=3 ; p<0,01\right)$.

\section{CUADRO 6}

Aceptación de diferentes métodos de control de la población de palomas. Los porcentajes no suman 100 ya que cada persona podía marcar varias opciones

\begin{tabular}{lcc} 
Método de control & $\begin{array}{c}\text { Porcentaje de personas que alimentan } \\
\text { a las palomas } n=161\end{array}$ & $\begin{array}{c}\text { Porcentaje de personas que no alimentan } \\
\text { a las palomas } n=90\end{array}$ \\
Anticonceptivos & 70 & 68 \\
Remoción de nidos & 19 & 51 \\
Repelentes & 21 & 32 \\
Ninguno & 22 & 11 \\
Depredadores & 11 & 32 \\
Control de alimento y agua & 6 & 30 \\
Esterilización & 4 & 11 \\
Traslado y educación & 2 & 9 \\
\hline
\end{tabular}

(Chi cuadrado de contingencia: $\left.X^{2}=36,55, g l=7 ; p<0,01\right)$.

\section{DISCUSIÓN}

Tradicionalmente la medida que más se ha recomendado para controlar las poblaciones de palomas son las campañas para no alimentarlas ya que al tener menos alimento disminuye su sobrevivencia y su potencial reproductivo. No obstante, esas campañas generalmente han fracasado o han tenido efecto solo mientras se aplica la campaña (Senar et al., 2016). Un ejemplo es un estudio realizado en Barcelona en el que se logró una reducción del $40 \%$ de la población, aunque aumentó nuevamente seis meses después de que se suspendió la campaña (Senar et al., 2016).
El bajo costo de establecer una venta informal y la ausencia de controles adecuados, permite que personas desempleadas y con baja escolaridad encuentren un sustento en esta actividad (Arámbulo III, Almeida, Cuéllar, \& Belotto, 1995; Trejos, 2000). Esto favorece que surjan hábitos difíciles de cambiar ya que se establecen intereses comerciales y afectivos que hacen que crezca la cantidad de palomas, de personas que venden comida para las palomas y de personas que las alimentan. Sin embargo, el hecho de que las personas tiendan a comprar alimento para aves cuando hay ventas informales cercanas de ese producto podría ser aprovechado para desarrollar una estrategia de manejo novedosa e interactiva con los 
visitantes del parque. Sería interesante determinar si el hecho de que las personas tiendan a comprar semillas para alimentar a las palomas cuando hay cerca ventas informales de esos productos se debe a que conocen la dieta natural de esas aves.

En Europa se han identificado cinco tipos de personas que alimentan a las palomas (Haag-Wackernagel, 1997): 1) los que Ilaman la atención de otros humanos; 2) los que quieren contacto social y forman redes de alimentadores de palomas; 3 ) los solitarios que alimentan a las palomas para compensar la falta de relaciones sociales; 4) los que por razones religiosas o éticas alimentan a las aves para que no mueran de hambre; y 5) los que se divierten alimentándolas. En nuestro caso, las categorías tres y cuatro se analizaron juntas como empatía y no identificamos personas que alimentaran a las palomas para llamar la atención ni para incorporarse a un grupo social. Contrariamente, encontramos un grupo importante de personas que las alimentan por tradición. Esta es una categoría nueva que debe ser analizada a nivel mundial para poder establecer estrategias de manejo. Esta categoría requiere una atención especial pues podría multiplicar la cantidad de personas que alimentan a las palomas.

Con frecuencia, las investigaciones sobre percepción de la naturaleza encuentran que las mujeres muestran mayor sensibilidad debido a que tienen mayor sentido de responsabilidad social que los hombres (Ryan, 2011; Durán, Barrientos, \& Charpentier, 2016), por lo que esperaríamos que alimentaran a las palomas principalmente por empatía. Sin embargo, encontramos que las razones son más variadas, en un futuro se debe analizar si esto se correlaciona con una mayor variedad de motivos por los que visitan los parques. Por el contrario, la empatía que sienten los hombres costarricenses por alimentar a las palomas puede tener razones culturales, de forma similar a lo que sucede con la tenencia de aves canoras en cautiverio (Johnes, 2007; Menacho-Odio \& Oviedo-Pérez, 2013).

Al igual que en otros estudios, encontramos que en general las personas que alimentan a las palomas tienen una percepción más positiva de las palomas y no las consideran una plaga (Ryan, 2011). Por otra parte, es probable que la indecisión sobre aceptar o no métodos de control radique en elementos de tipo ético en contra de la eutanasia, que ya han sido identificados en otros países (Ryan, 2011, Mondocorre et al., 2014, Ramírez-Alán, De la O-Castro, Bolaños-Picado, \& Queen-Blanco, 2017). El uso de anticonceptivos, es el método más aceptado, sin embargo, aunque hay casos exitosos como el de Ljubljana, Eslovenia, hay preocupación sobre cómo darles la dosis correcta, los efectos secundarios y el efecto en otras aves que pudieran tomar el anticonceptivo por error (HaagWackernagel, 1999; Dobeic, Pintarič, Vlahović, \& Dovč, 2011). El desarrollo de algunos inhibidores de la incubación como nicarbazina, han despertado interés por tener una capacidad parcial y temporal en la reducción de las poblaciones, pero se requieren más estudios (Giunchi et al., 2012). Por eso, antes de establecer una estrategia de manejo que incluya anticonceptivos o inhibidores de la incubación, recomendamos que se hagan más estudios sobre su uso y efectos.

La elaboración de una estrategia sostenible de manejo de la Paloma de Castilla debe incluir un plan de monitoreo y control de la salud de las palomas y un plan de educación continua que considere las razones por las que las personas alimentan a las palomas y la tendencia a comprar alimento apropiado para aves granívoras si hay ventas cercanas. Una estrategia que regule y gestione la venta de alimento y organice el suministro de sustancias anticonceptivas, en vez de prohibir la venta de alimento para palomas por completo, podría tener mejores resultados a largo plazo, pues se podría mantener a las aves saludables, regular el tamaño de la población, dar una fuente de ingreso a personas de escasos recursos y brindar una actividad atractiva para los visitantes de los parques.

\section{AGRADECIMIENTOS}

Las autoras agradecemos al público y a los funcionarios de las municipalidades de San José y Goicoechea y de la iglesia católica de Sabanilla que participaron en las encuestas. Sergio Quesada, Maribel Zúñiga y Carolina Seas brindaron apoyo técnico.

\section{REFERENCIAS}

Arámbulo III, P. V., Almeida, C. R., Cuéllar, J. A., \& Belotto, A. J. (1995). La venta de alimentos en la vía pública en América Latina. Boletín de la Oficina Sanitaria Panamericana (OSP), 118(2), 97-107. Recuperado de http://iris.paho.org/ xmlui/bitstream/handle/123456789/15621/v118n2p97. pdf? sequence $=1$ \&isAllowed $=y$

Artavia, S. (2017, 7 de octubre). 25 edificios patrimoniales urgen frenar deterioro ocasionado por palomas. San José, Costa Rica. Grupo Nación GN S.A. Recuperado de https://www.nacion.com/el-pais/patrimonio/25-edificios-patrimoniales-urgen-frenar-deterioro-ocasionado-por-palomas/NJIAPHVMOJAGRH7TMGTXGIC7IE/ story/

Arroyo, F. (2017, 8 de noviembre). Salud prohíbe actividades en parque de Guadalupe por culpa de las palomas. 
San José, Costa Rica. La Teja Grupo Nación GN S.A. Recuperado de: https://www.lateja.cr/nacional/salud-prohibe-actividades-en-parque-de-guadalupe/ XATPNZWKCJBNFPKVPWUEWSBLE4/story/

Astorga, L. (2019, 7 de junio). Palomas obligan a trasladar las paradas de autobús ubicadas en el parque de Guadalupe. San José, Costa Rica. La Nación Grupo Nación GN S.A. Recuperado de https://www.nacion.com/el-pais/ servicios/palomas-obligan-a-trasladar-las-paradas-de-autobus/5DX7DYMD4NFWDOW6PXXFBSXKF4/ story/

Cabezas, Y. (2013, 22 de octubre). Palomas cuestan caro a dueños de edificios y casas. San José, Costa Rica. CRHoy.com. Recuperado de https://archivo.crhoy.com/ palomas-cuestan-caro-a-duenos-de-edificios-y-casas/ nacionales/

Ciminari, M. E., Moyano, G., Chediack, J. G., \& Caviedes-Vidal, E. (2005). Feral pigeons in urban environments: dietary flexibility and enzymatic digestion? Revista Chilena de Historia Natural, 78(2), 267-279. DOI: 10.4067/ S0716-078X2005000200011

Dobeic, M., Pintarič, Š., Vlahović, K., \&Dovč, A. (2011). Feral pigeon (Columba livia) population management in Ljubljana. Veterinarskiarhiv, 81(2), 285-298. Recuperado de https:// www.cabi.org/ISC/FullTextPDF/2011/20113188849.pdf

Durán, M., Barrientos, Z., \& Charpentier, C. (2016). Percepción ambiental de escolares urbanos: influencia de áreas verdes, financiamiento y sexo en Costa Rica. UNED Research Journal, 8(1), 31-39. DOI: 10.22458/urj.v8i1.1220

Fernández, K. (2019, 21 de marzo). ¡Guácala! Máquinas de ejercicios en parque de Garantías Sociales están llenas de cuitas de paloma. San José, Costa Rica. La Teja Grupo Nación GNS.A. Recuperado de https://www.lateja.cr/nacional/guacala-maquinas-de-ejercicios-en-parque-de/ FUUF2H4FVJHHDC4AVNY4WLGZ7A/story/

Giunchi, D., Albores-Barajas, Y. V., Baldaccini, N. E., Vanni, L., \& Soldatini, C. (2012). Feral pigeons: problems, dynamics and control methods. En S. Soloneski (Ed.), Integrated pest management and pest control: current and future tactics, 215-240. Rijeka, Croacia: InTech. DOI: 10.5772/1383

Gutiérrez, F. (2010, 6 de abril). Cartagineses luchan contra las palomas de castilla. Cartago, Costa Rica. Al día Grupo Nación GN S.A. Recuperado de http://wvw.aldia.cr/ad_ ee/2010/abril/06/nacionales2325239.html

Haag-Wackernagel, D. (1997). Die soziokulturellen Ursachen des Taubenproblems. Deutsche Tierärztliche Wochenschrift, 104, 52-57. Recuperado de https://www. researchgate.net/profile/Daniel_Haag-Wackernagel/ publication/14061594_Sociocultural_reasons_for_the pigeon_problem/links/547de4bb0cf285ad5b08ae6d/ Sociocultural-reasons-for-the-pigeon-problem.pdf

Haag-Wackernagel, D. (1999). Feral Pigeon Management Regulation von Strassentaubenpopulationen. Recuperado de https://anatomie.unibas.ch/IntegrativeBiology/ haag/Feral-Pigeon-Strassentauben/pigeon-management.html\#Publikationen
Holdridge, L. R., Grenke, W. C., Hatheway, W. H., Liang, T., \& Tosi, J. A. (1971). Forest Environments in Tropical Life Zones: a Pilot Study. Oxford, U.K.: Pergamon Press.

Johnes, M. (2007). Pigeon racing and working-class culture in Britain, c. 1870-1950. Cultural and Social History, 4(3), 361-383. Recuperado de http://www.bbc.co.uk/ radio4/history/making_history/pigeon.pdf DOI: 10.2752/147800407X219250

La Nación. (2013, 12 de julio). Palomas del parque de Goicoechea murieron envenenadas con lacnate. San José, Costa Rica. La Nación Grupo Nación GN S.A. Recuperado de https://www.nacion.com/sucesos/seguridad/palomas-del-parque-de-goicoechea-murieron-envenenadas-con-lacnate/L4PKSXTAOJANZKD3AHA5544PU4/ story/

Lynch, D. (2018, 24 de enero). Darán alimento anticonceptivo a palomas para evitar sobrepoblación en Guadalupe y otros puntos. San José, Costa Rica. Teletica Televisora de Costa Rica S.A. Recuperado de https://teletica. com/184128_daran-alimento-anticonceptivo-a-palomas-para-evitar-sobrepoblacion-en-guadalupe-y-otros-puntos

Menacho-Odio, R. M., \& Oviedo-Pérez, P. (2013). Características culturales de la tenencia del jilguero (Myadestes melanops) en tres comunidades de Cartago. Biocenosis, 27(12), 44-49. Recuperado de http://investiga.uned.ac.cr/ revistas/index.php/biocenosis/article/view/598/497

Mondocorre, W., Pérez, F., Albis, N., \& Zeballos, M. (2014). Estimación de la abundancia de palomas (Columba livia) y percepción de la ciudadanía, para su control y manejo en la ciudad de Sucre. Ramos, M. (ed.), Ciencias Tecnológicas y Agrarias T-Il Handbook, 1-407. Sucre, Bolivia: USFX-Bolivia. Recuperado de https:// www.usfx.bo/nueva/Dicyt/Handbooks/Ciencias\%20 Tecnol\%C3\%B3gicas\%20y\%20Agrarias_2/Ciencias\%20 Tecnol\%C3\%B3gicas\%20y\%20Agrarias_Handbook_ Vol\%20II/PAPERS_25/art1.pdf

Monge-Nájera, J., Gómez, P., \& Rivas, M. (2008). Biodiversidad Tropical. San José, Costa Rica: EUNED.

Multimedios. (2018, 13 de diciembre). Darán anticonceptivos a las palomas de San José. San José, Costa Rica. Grupo Multimedios. Recuperado de https://www.multimedios.cr/nacional/ video-daran-anticonceptivos-las-palomas-de-san-jose

Municipalidad de Heredia. (2013). Sesión extraordinaria 2522013. Heredia, Costa Rica. Recuperado de https://www. heredia.go.cr/sites/default/files/sesion_extraordinaria_252-2013_acta1.pdf

Murton, R. K., \& Westwood, N. J. (1966). The foods of the rock dove and feral pigeon. Bird Study, 13(2), 130-146. DOI: $10.1080 / 00063656609476116$

Ramírez, O., Amador, M., Camacho, L., Carranza, I.J., Chaves, E., Moya, A., Vega, M., Verdesia, J., \& Quiros, W. (2008). Conocimiento popular de la Paloma de Castilla (Columba livia) en el Parque Central de Alajuela. Zeledonia, 12(1), 
14-19. Recuperado de https://www.zeledonia.com/uplo ads/7/0/1/0/70104897/2008-12-1.pdf

Ramírez-Alán, Ó., De la O-Castro, J., Bolaños-Picado, D., \& Queen-Blanco, J. K. Mc. (2017). Evaluación de la abundancia relativa y percepción de la presencia de palomas Columba livia (Columbiformes: Columbidae) en la Universidad Nacional de Costa Rica. Uniciencia, 31(1), 29-38. Recuperado de https://dialnet.unirioja.es/servlet/articulo?codigo=5817369 DOI: $10.15359 /$ ru.31-1.4

Repretel. (2016, 4 de noviembre). Nueva Plaza de la Cultura será libre de palomas. San José, Costa Rica: Representaciones Televisivas Repretel S.A. Recuperado de http://www.repretel.com/actualidad/ nueva-plaza--cultura-sera-libre--palomas-54088

Ryan, A. (2011). The distribution, density, and movements of feral pigeons Columba livia and their relationship with people (M.Sc. Thesis). Victoria University of Wellington, Wellington, NZ. Recuperado de http://researcharchive. vuw.ac.nz/handle/10063/2045

Senar, J. C., Montalvo, T., Pascual, J., \& Peracho, V. (2016). Reducing the availability of food to control feral pigeons: changes in population size and composition.
Pest management science, 73(2), 313-317. DOI: 10.1002/ ps.4272

Spennemann, D., \& Watson, M. (2017). Dietary habits of urban pigeons (Columba livia) and implications of excreta $\mathrm{pH}$ - a review. European Journal of Ecology, 3(1), 27-41. DOI: 10.1515/eje-2017-0004.

Stock, B., \& Haag-Wackernagel, D. (2014). Effectiveness of gel repellents on feral pigeons. Animals, 4(1), 1-15. DOI: 10.3390/ani4010001

Stringham, S. A., Mulroy, E. E., Xing, J., Record, D., Guernsey, M. W., Aldenhoven, J. T., Osborne, E. J., \& Shapiro, M. D. (2012). Divergence, convergence, and the ancestry of feral populations in the domestic rock pigeon. Current Biology, 22(4), 302-308. DOI: 10.1016/j.cub.2011.12.045

Trejos, M. E. (2000). La Cuestión Laboral en Costa Rica: Una Aproximación desde las Discusiones Actuales (pp. 135156). En: de la Garza Toledo, E. (ed.). Reestructuración Productiva, Mercado de Trabajo y Sindicatos en América Latina. Buenos Aires, Argentina: CLACSO. Recuperado de http://biblioteca.clacso.edu.ar/clacso/ gt/20101108025839/6trejos.pdf 